\title{
Improvement Of Block Product Turbo Coding By Using A New Concept Of Soft Hamming Decoder
}

\author{
Alaa Ghaith, PhD \\ HKS Laboratory, Electronics and Physics Dept., \\ Faculty of Sciences I, Lebanese University, Beirut, Lebanon \\ doi: 10.19044/esj.2016.v12n18p167 URL:http://dx.doi.org/10.19044/esj.2016.v12n18p167
}

\begin{abstract}
The block product turbo code (BPTC) is classified as one of block turbo code concatenation forms. The Hamming code can detect two-bit error and correct one-bit error. The BPTC uses two Hamming codes for "column" coding and "row" coding, it has improved the Hamming code correcting only one error. In addition, the BPTC carries out block interleaving coding for disorganizing the transmission sequence before transmission, so as to avoid burst errors when the signal meets multi-path channel in the channel. This paper will discuss the decoding mechanism of the BPTC and analyze the efficiency of using a soft decoding algorithm in the decoding process. The soft Hamming Decoder is based on error patterns which belong to the same syndrome. It is shown that it is sufficient to investigate error patterns with one and two errors to gain up to $1.2 \mathrm{~dB}$ compared to hard decision decoding. Here, we will consider also the error patterns with three errors which belong to the determined syndrome, which increases the gain and improves the quality of the soft-output due to the increased number of comparisons with valid code words, in despite that, it will increase the complexity of the decoding process. The system is based on two Hamming block channel code combinations, which can be similar or different, a block interleaving to construct a BPSK modulation and BPTC coding system in the concept of feedback encoding in turbo code over an AWGN channel. To observe its coding improvement, we present the simulation results for the soft decoding of the BPTC codes of a code word length from 49 bits (using two $(7,4)$ codes) up to 1440 bits (using two $(127,120)$ codes).
\end{abstract}

Keywords: Syndrome based soft decoding, Hamming Code, low complexity, soft-output, turbo decoding, BPTC, AWGN, block interleaver, coding gain, turbo code 


\section{Introduction}

Concatenated coding schemes were first proposed by Forney as a method for achieving large coding gains by combining two or more relatively simple block or component codes. The resulting codes had the error-correction capability of much longer codes, and they were endowed with a structure that permitted relatively easy to moderately complex decoding. A serial concatenation of codes is most often used for powerlimited systems. The most popular of these schemes consists of a ReedSolomon outer (applied first, removed last) code followed by a convolutional inner (applied last, removed first) code. A turbo code can be thought of as a refinement of the concatenated encoding structure plus an iterative algorithm for decoding the associated code sequence. Turbo codes were first introduced in 1993 by Berrou, and Glavieux, where a scheme is described that achieves a bit-error probability of $10^{-5}$ using a rate $1 / 2$ code over an AWGN channel and BPSK modulation at an $\mathrm{E}_{\mathrm{b}} / \mathrm{N}_{0}$ of $0.7 \mathrm{~dB}$. The codes are constructed by using two or more component codes on different interleaved versions of the same information sequence. Whereas, for conventional codes, the final step at the decoder yields hard-decision decoded bits (symbols), for a concatenated scheme such as a turbo code to work properly, the decoding algorithm should not limit itself to passing hard decisions among the decoders. To best exploit the information learned from each decoder, the decoding algorithm must effect an exchange of soft decisions rather than hard decisions. For a system with two component codes, the concept behind turbo decoding is to pass soft decisions from the output of one decoder to the input of the other decoder, and to iterate this process several times so as to produce more reliable decisions. The purpose was to find digital communications systems that have a capacity and a performance close to the limits found by Shannon. For applications that require error correcting codes to operate with much shorter delays, Berrou, Evano, and Battail have advocated block component codes, maintaining turbo coding decoding principle. These codes, called turbo- block codes, exhibit a coding gain that is considerably larger than that of the standalone component block codes. Moreover, the decoding complexity for these turbo-block codes is quite reasonable, as long as the decoding complexity of the component block codes is so. The block product turbo code (BPTC) is classified as one of block turbo code concatenation forms. The Hamming code can detect two-bit error or correct one-bit error. The BPTC uses two Hamming codes for "column" coding and "row" coding, it has improved the Hamming code correcting only one error. In addition, the BPTC carries out block interleaving coding for disorganizing the transmission sequence before transmission, so as to avoid burst errors. 
We will introduce here the same concept behind the turbo decoding which is to pass the soft decisions between both decoders, and to iterate this process several times to produce more reliable decisions. The major contributions of this work are presented by the proposition of a new soft decoding algorithm which can achieve a linear complexity with a small degradation compared to maximum likelihood decoding but it permits to us to consider the error patterns with three errors, and the utilization of this soft decoder in the turbo principal process which will provide a lot of gain when compared to the turbo hard decision decoder and to the single soft decoder.

The remainder of this paper is organized as follows. In Section II, we discuss the background about the Hamming codes. Section III presents the syndrome based soft, and the soft decoding technique. The system design of the BPTC coding and decoding schemes are also presented in Section IV. The system performance is investigated in Section $\mathrm{V}$ through extensive trace-driven simulation. Finally, conclusions are given in Section VI along with the suggestions for future work.

\section{Theoretical Background}

A. Encoding and Transmission

The encoding of the message bits $\boldsymbol{m}$ can be performed by a modulo 2 vector matrix multiplication of $\boldsymbol{m}$ and the generator matrix $\boldsymbol{G}$

$$
\boldsymbol{c} \equiv \boldsymbol{m} \cdot \boldsymbol{G}
$$

The expression " $\equiv "$ is equivalent with $\boldsymbol{c}=(\boldsymbol{m} . \boldsymbol{G})$ modulo 2 .

Hamming code is an important forward error correction (FEC) in theory and practice so far. It is a sort of binary linear block code. It put forward an important single-error-correcting code, using parity check matrix $(\mathrm{H})$ to detect and correct errors. It is a simple type of systematic code, described as the following structure.

Block length: $n=2^{p}-1$

Number of data bits: $k=2^{p}-p-1$

Number of check bits: $n-k=p$

Minimum distance: $d_{\min }=3 \rightarrow$ Correct single bit error

$$
(n, k)=\left(2^{p}-1,2^{p}-1-p\right)
$$

We can use the following $k \times n$ array to define the generator matrix $(G)$

$$
G=\left[\begin{array}{c}
V_{1} \\
V_{2} \\
\vdots \\
V_{k}
\end{array}\right]=\left[\begin{array}{cccc}
V_{11} & V_{12} & \cdots & V_{1 n} \\
V_{21} & V_{22} & \cdots & V_{2 n} \\
\vdots & \vdots & \cdots & \vdots \\
V_{k 1} & V_{k 2} & \cdots & V_{k n}
\end{array}\right]
$$

Among which, $V_{1} \sim V_{k}$ are linearly independent vectors that can generate all code vectors. The data of the transmitting terminal are usually 
expressed in column vectors, therefore, the sequence of $\mathrm{k}$ message bits, i.e. the message $\boldsymbol{m}$ is expressed as $1 \times k$ matrix.

The generator matrix of systematic $(7,4)$-Hamming code is given by

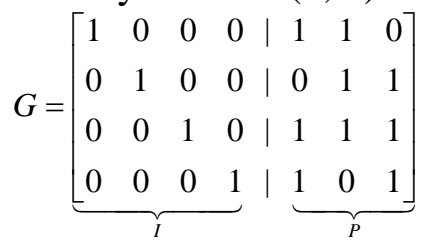

After the encoding, $\boldsymbol{c}$ is modulated, so that a logical zero is equivalent to $\mathrm{a}+1$ and a logical one is equivalent to $\mathrm{a}-1, x \in\{ \pm 1\}$. The modulated signal $\boldsymbol{x}$ is distorted by the additive white Gaussian noise (AWGN) $\boldsymbol{w}$ and results in the receive signal $y$,

$$
\boldsymbol{y}=\boldsymbol{x}+\boldsymbol{w}
$$

B. Hard Decision Decoding

In order to decode the received signals, we need to define a parity check matrix and a syndrome. There is a $(n-k) \times n$ matrix $\boldsymbol{H}$ in each generator matrix $\boldsymbol{G}$, so that the columns of $\boldsymbol{G}$ are orthogonal to the columns of $\boldsymbol{H}$, i.e. $\boldsymbol{G} \cdot \boldsymbol{H}^{T}=0$, the $\boldsymbol{H}^{T}$ is the transpose matrix of $\boldsymbol{H}$. In order to meet the orthogonality of system coding, the component of matrix $\boldsymbol{H}$ can be expressed as $H=\left[I_{n-k} \mid P^{T}\right]$

Therefore, the matrix $\boldsymbol{H}^{T}$ can be expressed as

$$
H^{T}=\left[\frac{I_{n-k}}{P^{T}}\right]
$$

$\boldsymbol{c}$ is the code word derived from matrix $\boldsymbol{G}$ if and only if $\boldsymbol{c} \cdot \boldsymbol{H}^{T}=0$. Let $\boldsymbol{r}$ be the vector received by the receiving terminal, so the $\boldsymbol{r}$ can be described as $\boldsymbol{r}=\boldsymbol{c}+$ $\boldsymbol{e}$, among which, $\boldsymbol{e}$ is the error vector resulted from the channel. The syndrome is defined as $\boldsymbol{s}=\boldsymbol{r} . \boldsymbol{H}^{T}$, it is the result of parity check implemented in $\boldsymbol{r}$, judge whether $\boldsymbol{r}$ is an effective element in the codeword set. Based on development of equation $\boldsymbol{s}=(\boldsymbol{c}+\boldsymbol{e}) \cdot \boldsymbol{H}^{T}=\boldsymbol{c} \cdot \boldsymbol{H}^{T}+\boldsymbol{e} \cdot \boldsymbol{H}^{T}$. However, for all elements in codeword set $\boldsymbol{c} \cdot \boldsymbol{H}^{T}=0$, therefore $\boldsymbol{s}=\boldsymbol{e} \cdot \boldsymbol{H}^{T}$.

Since the correction capability of Hamming code is 1 , meaning the error pattern is one selected from $\boldsymbol{n}$. Error patterns with 2 (duets) or 3 errors (triplets) which belong to the same syndrome are not taken into account for the decoding and the distorted code word is corrected as follow:

1.Use $\boldsymbol{s}=\boldsymbol{r} . \boldsymbol{H}^{T}$ to calculate the syndrome of $\boldsymbol{r}$

2.Find out common first item (error pattern) $\boldsymbol{e}_{j}$, its syndrome equals $\boldsymbol{r} . \boldsymbol{H}^{T}$

3.This error pattern is supposed to be the error caused by channel

4. The identified receive correction vector or code word equals $\boldsymbol{c}=\boldsymbol{r}+\boldsymbol{e}_{j}$

In fact, every double error is decoded to a valid but wrong code word. This explains the poor performance of HDD for Hamming codes. 


\section{Soft-Output Decoding}

\section{A. Syndrome Based Soft Decision Decoding}

For the syndrome based soft decision decoding it is required to calculate the log-likelihood ratios (LLR) from the received signal $y$,

$$
L(x \mid y)=\ln \frac{P(x=+1 \mid y)}{P(x=-1 \mid y)}
$$

which finally leads to

$$
L(x \mid y)=\ln \frac{\exp \left(-\frac{E_{b}}{N_{0}}(y-1)^{2}\right)}{\exp \left(-\frac{E_{b}}{N_{0}}(y+1)^{2}\right)}=\frac{4 E_{b}}{N_{0}} y,
$$

assuming that a logical zero and one have the same probability.

Let us assume that the syndrome of the distorted bit sequence of a $(7,4)$ Hamming code is $\boldsymbol{s}=\left(\begin{array}{lll}0 & 0 & 1\end{array}\right)$. The possible error patterns are collected in matrix $\boldsymbol{E}$ with its elements $\boldsymbol{e}_{j, i}$

$$
E=\left(\begin{array}{lllllll}
0 & 0 & 0 & 0 & 0 & 0 & 1 \\
\hline 1 & 0 & 1 & 0 & 0 & 0 & 0 \\
0 & 1 & 0 & 0 & 0 & 1 & 0 \\
0 & 0 & 0 & 1 & 1 & 0 & 0 \\
\hline 1 & 1 & 0 & 0 & 1 & 0 & 0 \\
1 & 0 & 0 & 1 & 0 & 1 & 0 \\
0 & 1 & 1 & 1 & 0 & 0 & 0 \\
0 & 0 & 1 & 0 & 1 & 1 & 0
\end{array}\right)
$$

where the second to fourth row bears the duets and the fifth to eighth row bears the triplets. The error patterns for all syndromes are determined in advance and stored in a list. The size of the list rises quadratically for double errors and cubically for triple errors. Every row of $\boldsymbol{E}$ is multiplied by the absolute value of LLRs of the received signal $L(x \mid y)$. Afterwards, the resulting row vector is added up. The vector with the lowest sum of LLR suggests the error pattern with the highest probability of a correct decoding.

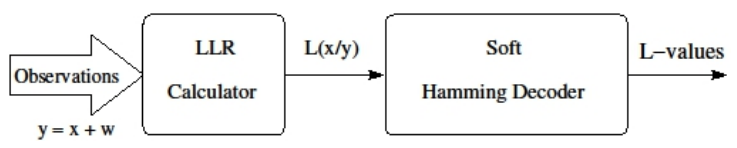

Fig. 1: Soft-Output Hamming Decoder

\section{B. Soft Decoding}

The structure of the soft hamming decoder is shown in Fig. 1. In general, soft-output decoding provides output values for iterative or turbo decoding. In order to generate soft-outputs, the following algorithm is proposed. The probability values of a code word are given by 


$$
P\left(\hat{c}_{j}=c_{j} \mid y_{j}\right)=\frac{\exp \left(\left|L\left(x_{j} \mid y_{j}\right)\right|\right)}{1+\exp \left(\left|L\left(x_{j} \mid y_{j}\right)\right|\right)}
$$

Next, the probability values are multiplied column-wise for the given error pattern of every row $i$.

$$
\tilde{P}_{i}=\prod_{j} \tilde{P}_{j} \text { where } \tilde{P}_{j}=\left\{\begin{aligned}
P\left(\hat{c}_{j}=c_{j} \mid y_{j}\right) & \text { if } e_{i, j}=0 \\
1-P\left(\hat{c}_{j}=c_{j} \mid y_{j}\right) & \text { if } e_{i, j}=1
\end{aligned}\right.
$$

Now $\tilde{P}_{i}$ is normalized, so that the sum of the normalized probabilities $P_{i}$ over all rows $i$ is equal to $1, \sum_{i} P_{i}=1$. So the probabilities $P_{i}$ are given by

$$
P_{i}=\frac{\widetilde{P}_{i}}{\sum_{i^{\prime}} \widetilde{P}_{i^{\prime}}}
$$

$P_{i}$ can be interpreted as the probability of correct decoding for the given error pattern of row $i$. In a last step, the probability that $x_{j}=+1$, for a given received code word $\boldsymbol{y}$, is calculated by the sum of $P_{i}$ over all rows $i$, if $\boldsymbol{e}_{i, j}=$ $\hat{c}_{j}$, where $\hat{c}_{j}$ is defined as the logical received bit sequence. The estimation of the new probabilities after the soft decoding.

$$
\hat{P}\left(x_{j}=+1 \mid y\right)=\sum_{\substack{i \\ e_{i, j}=\hat{c}_{j}}} P_{i}
$$

Due to the normalization, so that $\sum_{i} P_{i}=1$, the probability of $\hat{P}\left(x_{j}=-1 \mid y\right)$ can be calculated by

$$
\hat{P}\left(x_{j}=-1 \mid y\right)=1-\hat{P}\left(x_{j}=+1 \mid y\right)
$$

In order to exchange the information for turbo decoding it is required to calculate L-values from the derived probabilities.

\section{Some Simulation Results}

For the simulation results of the soft decoding, hamming codes of a code word length for 7 till 127 bit were investigated. Fig. 2, 3, 4, 5, and 6 illustrate the performance of the different decoding strategies for a certain code word length. Tab. 1 summarizes the results for all non-iterative codes. 

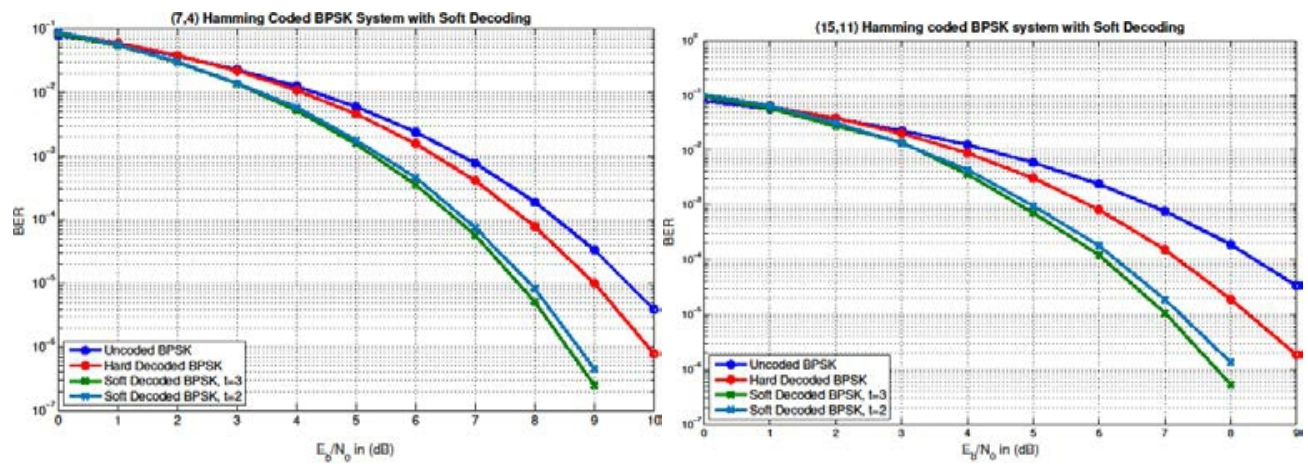

Fig. 2: BER for different decoding for a $(7,4)$ and $(15,11)$-Hamming code

Fig. 2 shows the bit error rate of the $(7,4)$-Hamming code for different types of decoding. It is shown that the decoding performance of the duet and triplet decoding is quite similar and very close to the union bound which is an upper bound for the bit error probability after maximum likelihood decoding. For the evaluation we focus on a $\mathrm{BER}=10^{-4}$. The coding gain amounts to $0.31 \mathrm{~dB}$ for the HDD, $1.36 \mathrm{~dB}$ for the duet decoding, and $1.46 \mathrm{~dB}$ for the triplet decoding. The extension of the code word length, up to $15 \mathrm{bit}$, results in a further performance gain. It is also apparent that the difference between duet decoding and triplet decoding rises. The coding gain amounts to $0.9 \mathrm{~dB}$ for the HDD and $1.8 \mathrm{~dB}$ for the duet decoding. Further $0.25 \mathrm{~dB}$ can be gained by triplet decoding.

\begin{tabular}{|rr|rr|rr|rl|}
\hline $\mathrm{N}_{\mathrm{c}}$ & $\mathrm{K}_{\mathrm{c}}$ & HDD & Gain & Duets & Gain & Triplets & Gain \\
\hline 7 & 4 & 7.80 & 0.31 & 6.75 & 1.36 & 6.65 & 1.46 \\
\hline 15 & 11 & 7.20 & 0.90 & 6.30 & 1.80 & 6.05 & 2.05 \\
\hline 31 & 26 & 7.00 & 1.21 & 6.05 & 2.16 & 5.80 & 2.41 \\
\hline 63 & 57 & 7.00 & 1.21 & 6.05 & 2.16 & 5.75 & 2.46 \\
\hline 127 & 120 & 7.10 & 1.11 & 6.35 & 1.86 & 6.00 & 2.21 \\
\hline
\end{tabular}

Tab. 1: Simulation Results for $\mathrm{E}_{\mathrm{b}} / \mathrm{N}_{0}$ in $\mathrm{dB}$ for a $\mathrm{BER}=10^{-4}$, and the resulting coding gain (uncoded $8.11 \mathrm{~dB}$ for $\mathrm{BER}=10^{-4}$ )

The $(31,26)$-Hamming code obtained the results for codes, for duets as well as for triplets. Fig. 3 shows that the coding gain amounts to $2.16 \mathrm{~dB}$ for the duet decoding and $2.41 \mathrm{~dB}$ for the triplet decoding. Same figure can be drawn for the $(63,57)$-Hamming code. The coding gain is similar to the (31,26)-Hamming code, but the bit error curve falls sharply. It is also shown that the difference between duet and triplet decoding is higher with $0.3 \mathrm{~dB}$. 

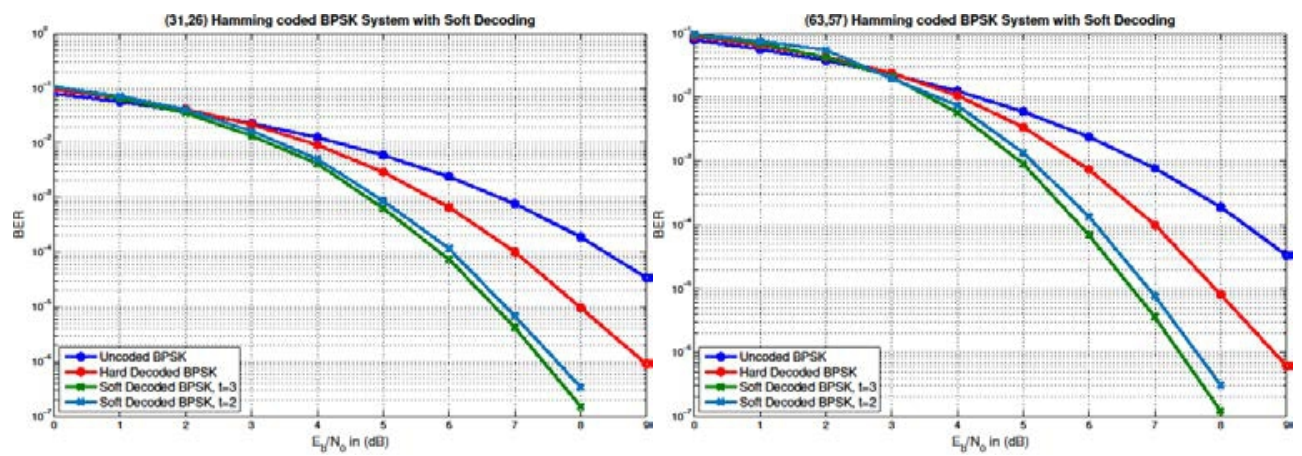

Fig. 3: BER for different decoding for a $(31,26)$ and $(63,57)$-Hamming code

Quite a similar picture can be drawn for the $(127,120)$-Hamming code which has the highest code rate $(\mathrm{Rc}=0.944)$ of the considered Hamming codes (see Fig. 4). The coding gain is lower than for the $(31,26)$ Hamming code (1.86 dB gain for the duet and $2.21 \mathrm{~dB}$ gain for the triplets), but the bit error curve falls more sharply. It is also shown that the difference between duet and triplet decoding is the highest with $0.35 \mathrm{~dB}$.

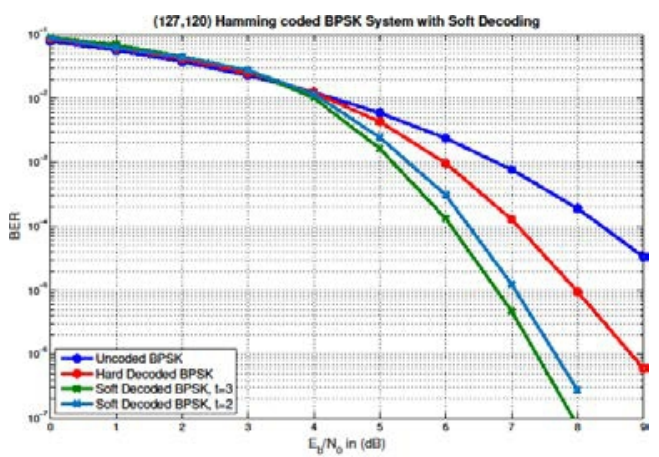

Fig. 4: BER for different decoding for a $(127,120)$-Hamming code

\section{BPTC Coding and Decoding Schemes}

Hamming put forward an important error-correcting code, Hamming code in 1948. It uses parity check matrix $(\mathrm{H})$ to detect and correct errors, however, its ability in detection and correction is limited, it can only detect 2-bit errors or correct 1-bit errors.

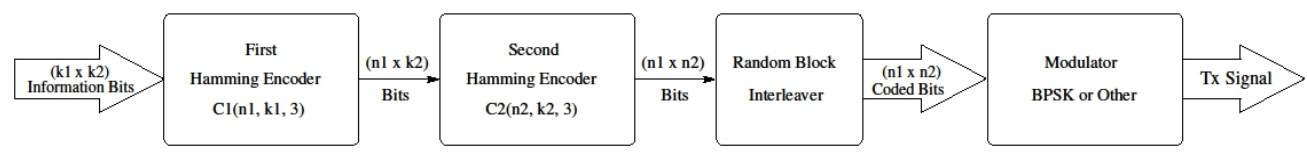

Fig. 5: BPTC Encoder

The block product turbo code (BPTC) is classified as one of block turbo code concatenation forms. The Hamming code can detect two-bit error 
or correct one-bit error. The BPTC uses two Hamming codes for "column" coding and "row" coding, it has improved the Hamming code correcting only one error. The encoder starts with the first row of information bits, calculates and appends the parity bits, and then moves on to the second row. This is repeated for each row. Next, the encoder starts with the first column of information bits, calculates and appends the parity bits for that column, and moves to the next column. Once the information block is complete, the encoder calculates and appends parity bits onto rows. It is important to note that different code lengths may be used for the horizontal and vertical blocks. In addition, the BPTC carries out block interleaving coding for disorganizing the transmission sequence before transmission, so as to avoid burst errors.

\section{A. Encoder System Design}

The general structure of a BPTC encoder is shown in Fig. 5. It consists of two systematic hamming encoders $C_{1}$ and $C_{2}$. It should be noted that the size of these two hamming codes could be the same and the free distance of any hamming code is always 3 which means it can correct one-bit error. The output sequences, however, are the same for identical input sequences. The $N$ bit data block is first arranged in $\left(k_{1} \times k_{2}\right)$ matrix form before encoded by $C_{1}$, an additional zero padding bits are placed at the end of the data block if needed. After encoded by the first encoder, the output block is then $\left(n_{1} \times k_{2}\right)$ matrix after adding the corresponding bits to each column. The output data block of the $C_{1}$ is also encoded by $C_{2}$ giving an output encoded data of $\left(n_{1} \times n_{2}\right)$ matrix after adding the corresponding bits to each row. Then this data block will be interleaved by a random interleaver. The main purpose of the interleaver is to randomize bursty error patterns so that it can be correctly decoded. It also helps to increase the minimum distance of the BPTC. The turbo coder obtained here can be described with the following structure.

Block length: $N=n_{1} \times n_{2}$

Number of data bits: $K=k_{1} \times k_{2}$

Number of check bits: $P=\left(n_{1}-k_{1}\right) \times k_{2}+\left(n_{2}-k_{2}\right) \times k_{1}+k_{1} \times k_{2}$

Coding Rate: $R=K / N=R_{1} \times R_{2}$.

\section{B. Decoder System Design}

The decoding procedure described below is generalized by cascading elementary decoders illustrated in Fig. 6. Let us consider the decoding of the rows and columns of a product code described in Section A and transmitted on a Gaussian channel using BPSK signaling. On receiving $\left(n_{1} \times n_{2}\right)$ matrix $\mathrm{R}$ corresponding to a transmitted $\left(k_{1} \mathrm{x} k_{2}\right)$ codeword $\mathrm{E}$, the second decoder, corresponding to the second encoder $C_{2}$, performs the decoding of the rows using the input matrix but first de-interleaved by the block de-interleaver corresponding to the interleaver used at the transmitter. The output $\left(n_{1} \times k_{2}\right)$ 
matrix of the second decoder is entered to the first decoder, corresponding to the first encoder $C_{1}$, which performs the decoding of the columns.

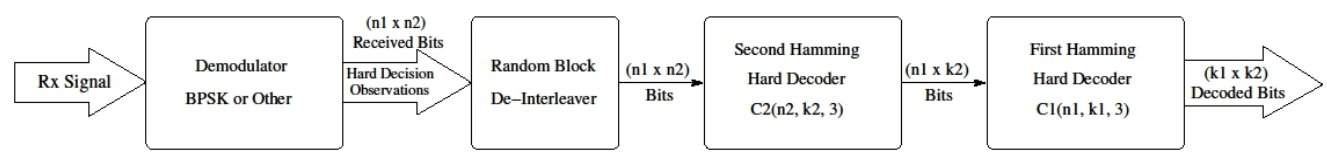

Fig. 6: BPTC Hard Decoder

\section{BPTC coding mechanism analysis}

Although the BPTC is composed of Hamming code, its probability of being corrected in the second dimension coding is increased by using the fundamental characteristics of turbo code. So the correction capability will increase normally to more than three-bits error. But we should mention here that it is unfair to compare a simple hamming code with the corresponding product turbo code formed by the concatenation of two from this simple code. For example, we can't compare the performance of the $(7,4)$-Hamming code with the BPTC code formed from two concatenated $(7,4)$-Hamming code, because the first one has a coding rate of 4/7 slightly bigger than half, but the second one has a coding rate of $(4 / 7)^{2}$ slightly lower than third. Therefore, in the simulation results presented here we will consider these remark by comparing approximatively equally coding rate.

Fig. 7 shows the performance of a BPSK system aver AWGN channel using a BPTC coding with the conventional hard decoding. We can simply remark that a gain is obtained with respect to the hard decoding of a simple hamming coding at high SNR (bigger than $5 \mathrm{~dB}$ ). And the curves of BPTC system are sharper when the codeword length of the coding used increase. Also the different curves in Fig. 7 show that at approximatively 6.3 to $6.5 \mathrm{~dB}$ we can obtain a BER $=10^{-4}$. Finally, these results show that the proposed Soft decoder of a simple Hamming decoder, where we can obtain the $10^{-4}$ of BER at approximatively $6.05 \mathrm{~dB}$ (see Tab. 1), can give a better performance than most of the Hard decoded BPTC system, and the gain is around 0.25-0.3 dB (see Fig. 2, 3, and 4).
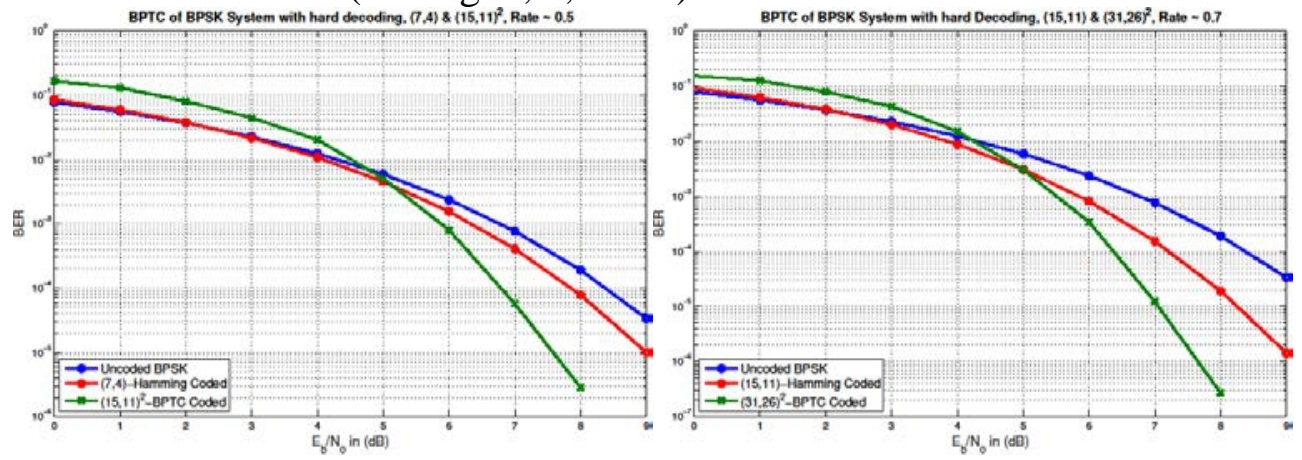

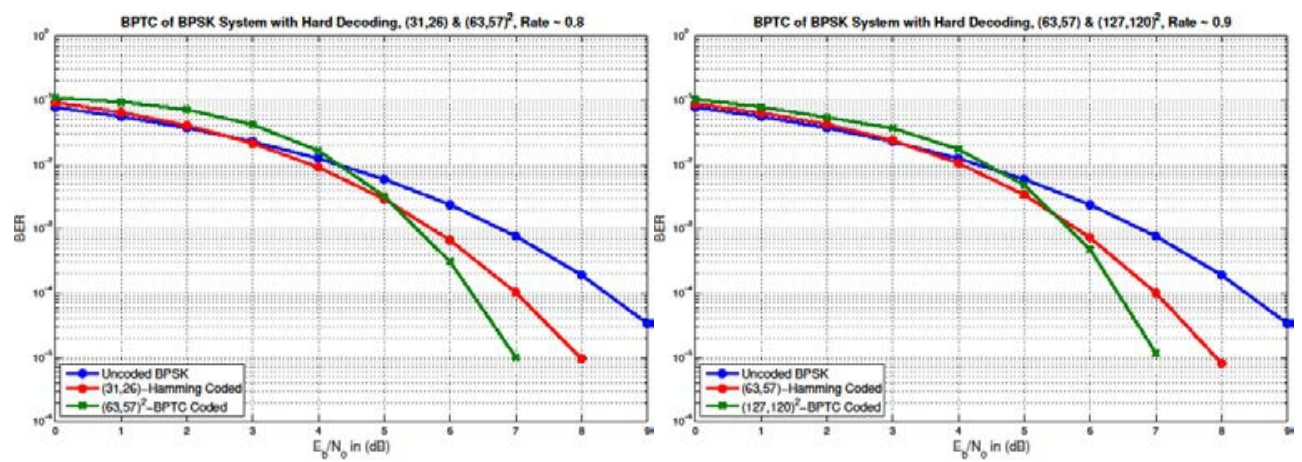

Fig. 7: BER of BPTC for different rates with Hard Decoding

\section{BPTC with Soft Decoder}

The LLR based decoding procedure described above can be used in the Soft Decoder of the BPTC. The Decoding process is done by cascading the proposed decoders and it is illustrated in Fig. 8. Let us consider the soft decoding of the rows and columns of a product code described in Section A and transmitted on a Gaussian channel using BPSK signaling. On receiving the observations $y$ corresponding to the message $x$ transmitted. The LLR calculator compute the $\left(n_{1} \times n_{2}\right)$ L-values matrix corresponding to these observations, after the block de-interleaver, the second soft decoder performs the decoding of the rows using the input LLR matrix to compute the $\left(n_{1} \times n_{2}\right)$ L-values output. Only the $\left(n_{1} \times k_{2}\right)$ portion of the output matrix is taking into account in the first soft decoder which performs the soft decoding of the columns and give at its output $\left(n_{1} \times k_{2}\right)$ containing the $\left(k_{1} \times k_{2}\right)$ L-values corresponding to the sent codeword. Finally, a threshold based decision device is needed to obtain the $\left(k_{1} \times k_{2}\right)$ output decoded bits.

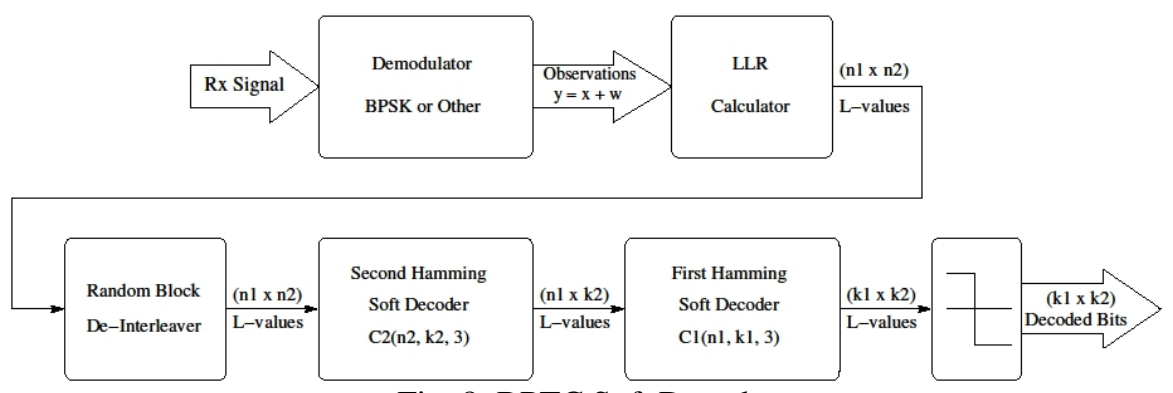

Fig. 8: BPTC Soft Decoder

\section{Simulation Results and Analysis}

A key performance index to evaluate the capacity-approaching is the BER given a received SNR over an AWGN channel. We consider a received SNR from 0 to $9 \mathrm{~dB}$ and examine the BER, as shown in the performance figures presented previously, Fig. 2, 3, 4, and 7. The received signal to noise 
ratio is considered here as $\mathrm{E}_{\mathrm{b}} / \mathrm{N}_{0}$ where $\mathrm{E}_{\mathrm{b}}$ is the received energy per bit, and $\mathrm{N}_{0}$ is the noise power spectral density. Monte Carlo simulation by MatLab is used to obtain the results shown in the past and following figures. For the evaluation, all size of hamming code from code length 7 into 127 was compared together, and the value of coding rate of each product turbo code is considered when compared to a simple hamming code. The code chosen are the $(7,4),(15,11),(31,26),(63,57)$, and $(127,120)$-Hamming code. And the turbo product code chosen are formed by to identical hamming code. We can predict before simulations that the BPTC formed by the $(7,4)$-Hamming code will not give good performances because its small code length.

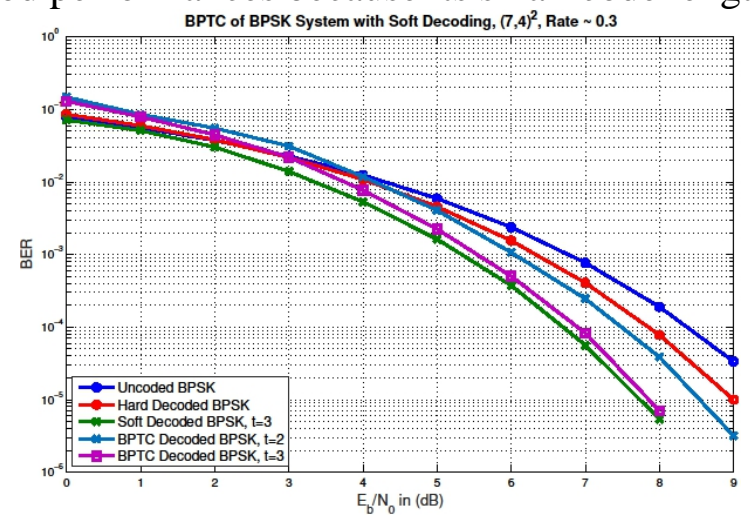

Fig. 9: Simple (7,4)-Hamming code vs. BPTC.

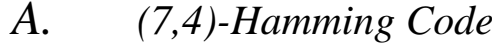

For the $(7,4)$-Hamming code, which takes 4 information bits and adds 3 parity bits to give the corresponding codeword and has approximatively half coding rate. The concatenation of two $(7,4)$-Hamming code which form the $(7,4)^{2}$ BPTC will encode 16 information bits into 49 coded bits and will have a coding rate near to 0.3. Normally, the BPTC should give a better performance than the simple hamming code. But Fig. 9 shows, in despite that the soft decoding of the simple hamming code improve the performance of hard decoding by approximately $1 \mathrm{~dB}$ at BER $=10^{-4}$, the performance of the BPTC with soft decoder decrease this gain to $0.9 \mathrm{~dB}$. This is slightly predictable because of the small size of this code. So, it is un-useful to concatenate the $(7,4)$-Hamming code.

B. (15,11)-Hamming Code

For the (15,11)-Hamming code, which gives to each 11 information bits 4 additional parity bits, and has a coding rate of approximately 0.7 . The $(15,11)^{2}$ BPTC formed by the concatenation of these two hamming will encode each 121 information bits to 225 coded bits and will have approximately half code rate. Fig. 10 shows also that the performance of the BPTC using the soft decoder which correct duets bit error. Using the soft 
decoder correcting the triplets bit error, the BPTC increases the gain of the simple hamming code soft decoded by approximately $0.5 \mathrm{~dB}$ and the gain with the hard decoded to $1.5 \mathrm{~dB}$ at $\mathrm{BER}=10^{-4}$.

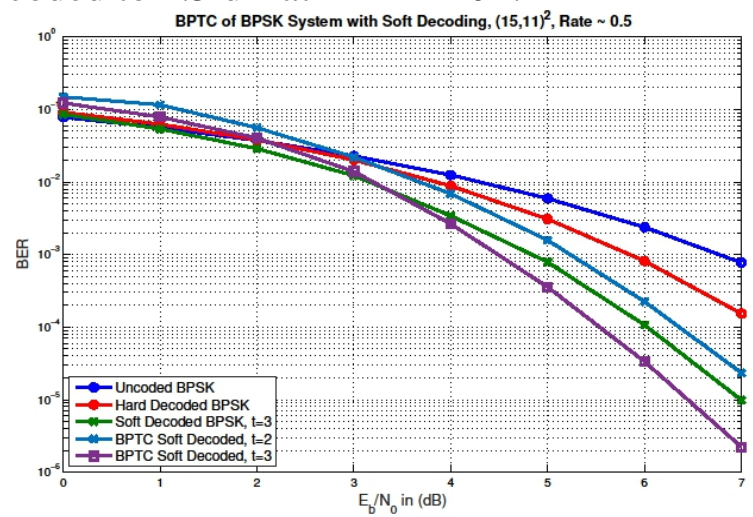

Fig. 10: Simple (15,11)-Hamming code vs. BPTC.

\section{C. (31,26)-Hamming Code}

The $(31,26)$-Hamming code encodes every 26 information bits into 31 coded bits, thus, its coding rate is 0.8 . However, the $(31,26)^{2}$ BPTC formed by the concatenation of two of this hamming code has a coding rate approximately equal to 0.7 where it encodes each 676 information bits into 961 coded bits. The results, presented in Fig. 11, show a big improvement of the BPTC using soft decoder of triplet error bit with respect to the soft decoding of a simple Hamming code, where it increases the gain to $0.75 \mathrm{~dB}$ at $\mathrm{BER}=10^{-4}$, and to $2 \mathrm{~dB}$ with respect to the hard decoding.

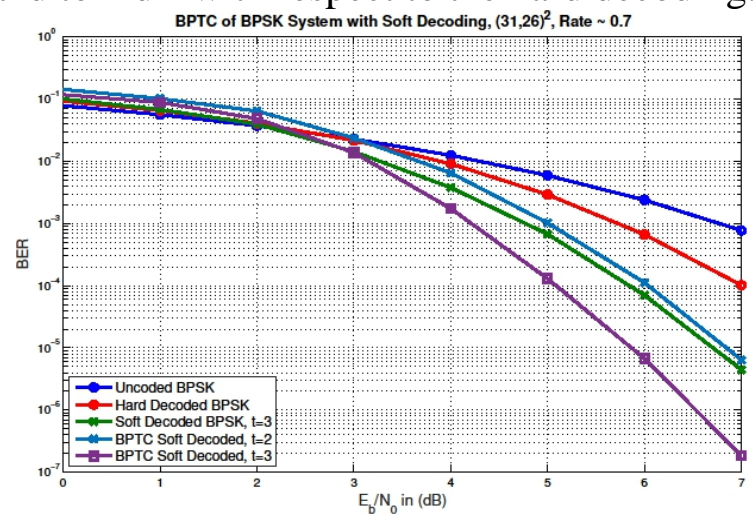

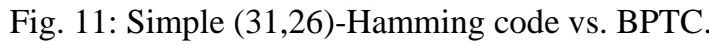


D.

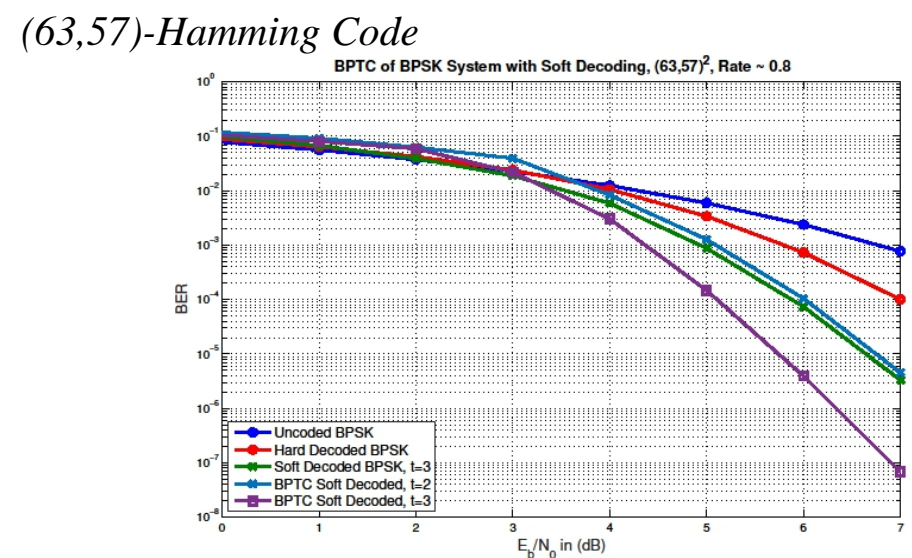

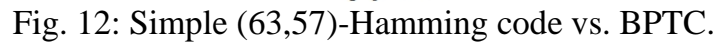

The (63,57)-Hamming code encodes every 57 information bits into 63 coded bits, so, its coding rate is 0.9 . However, the $(63,57)^{2}$ BPTC formed by the concatenation of two of $(63,57)$-Hamming code has a coding rate equal to 0.8 where it encodes each 3249 information bits into 3969 coded bits. Fig. 12 shows that the gain between the BPTC using soft decoder of triplet error bit and the soft decoding of a simple Hamming code is slightly decreased from $0.75 \mathrm{~dB}$ in the previous case to $0.72 \mathrm{~dB}$ here at BER $=10^{-4}$, in despite that the gain with respect to the hard decoding remains the same (2 $\mathrm{dB})$.

E. (127,120)-Hamming Code

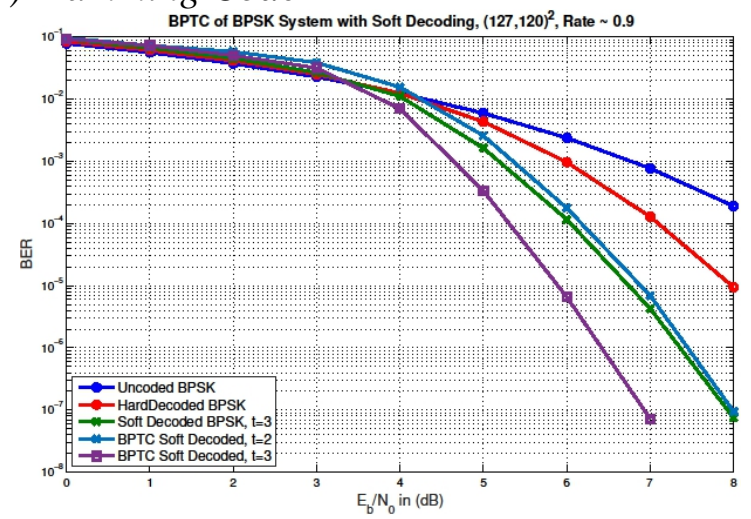

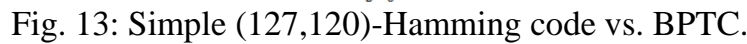

The same phenomenon is remarked in the Fig. 13, where we compared the $(127,120)$-Hamming code, which encodes 120 information bits into 127 coded bits with a rate equal to 0.94 , with the $(127,120)^{2}$ BPTC code, which is formed by the concatenation of both of this code to obtain a code rate approximately equal to 0.9 . Thus the improvement is decreased to 0.7 
$\mathrm{dB}$ at $\mathrm{BER}=10^{-4}$ with respect to the soft decoding of the simple Hamming code and to $1.7 \mathrm{~dB}$ when compared to the hard decoding.

\section{F. $\quad$ BPTC Comparison}

With respect to the performances shown in the last five figures (Fig. 9, 10, 11, 12, and 13), the question will be: "Which is the better BPTC code to use?” Fig. 14 shows a comparison between the five cases presented before, where we remark directly that the $(7,4)^{2}$ case is the worst case, so it gets out from the competition. The $(31,26)^{2}$ and the $(63,57)^{2}$ cases show the best performance, where the $(31,26)^{2}$ BPTC slightly outperforms the $(63,57)^{2}$ BPTC at low SNR $(\mathrm{SNR}<5 \mathrm{~dB})$ and the $(63,57)^{2}$ BPTC has a very small gain with respect to the $(31,26)^{2}$ BPTC code. We should not forget the coding rate when we want to compare different schemes of coding.

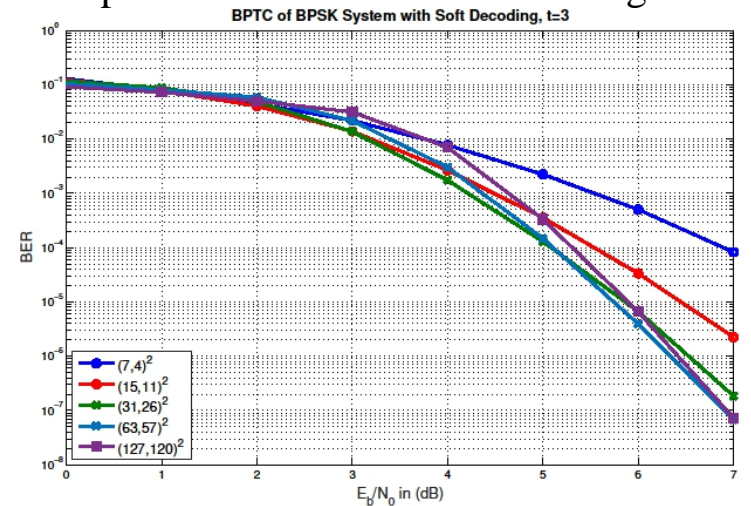

Fig. 14: BPTC performances comparison.

The Fig. 15 shows the performances of the block product turbo code using hamming and the performances of the simple hamming code which has approximately the same corresponding coding rate. Both use the soft decoding algorithm presented previously. The results obtained show that the BPTC outperforms the simple hamming code in all cases and rates. For example, let us see the half rate case, where we compare the $(15,11)^{2}$ to $(7,4)$-Hamming code, the gain obtained here is about $1 \mathrm{~dB}$ at $\mathrm{BER}=10^{-4}$. When the coding rate is 0.7 in the case of the $(31,26)^{2}$ and the $(15,11)$ Hamming code, the gain obtained is also about $1 \mathrm{~dB}$ at $\mathrm{BER}=10^{-4}$. This gain will be decreased slowly when we compare the $(63,57)^{2}$ and the $(31,26)$ Hamming code, where the coding rate is equal to 0.8 , and it will be equal to $0.75 \mathrm{~dB}$ at $\mathrm{BER}=10^{-4}$. The decreasing of gain continues with the last case where the coding rate is approximately 0.9 , the $(127,120)^{2}$ compared to the $(63,57)$-Hamming code, and this gain is decreased to $0.6 \mathrm{~dB}$ at $\mathrm{BER}=10^{-4}$. We should mention, finally, that the $(31,26)^{2}$ BPTC code has the best performance at $\mathrm{BER}=10^{-4}$ where it obtained it at $5 \mathrm{~dB}$ with a gain of 0.25 $\mathrm{dB}$ to the nearest block product turbo code. 

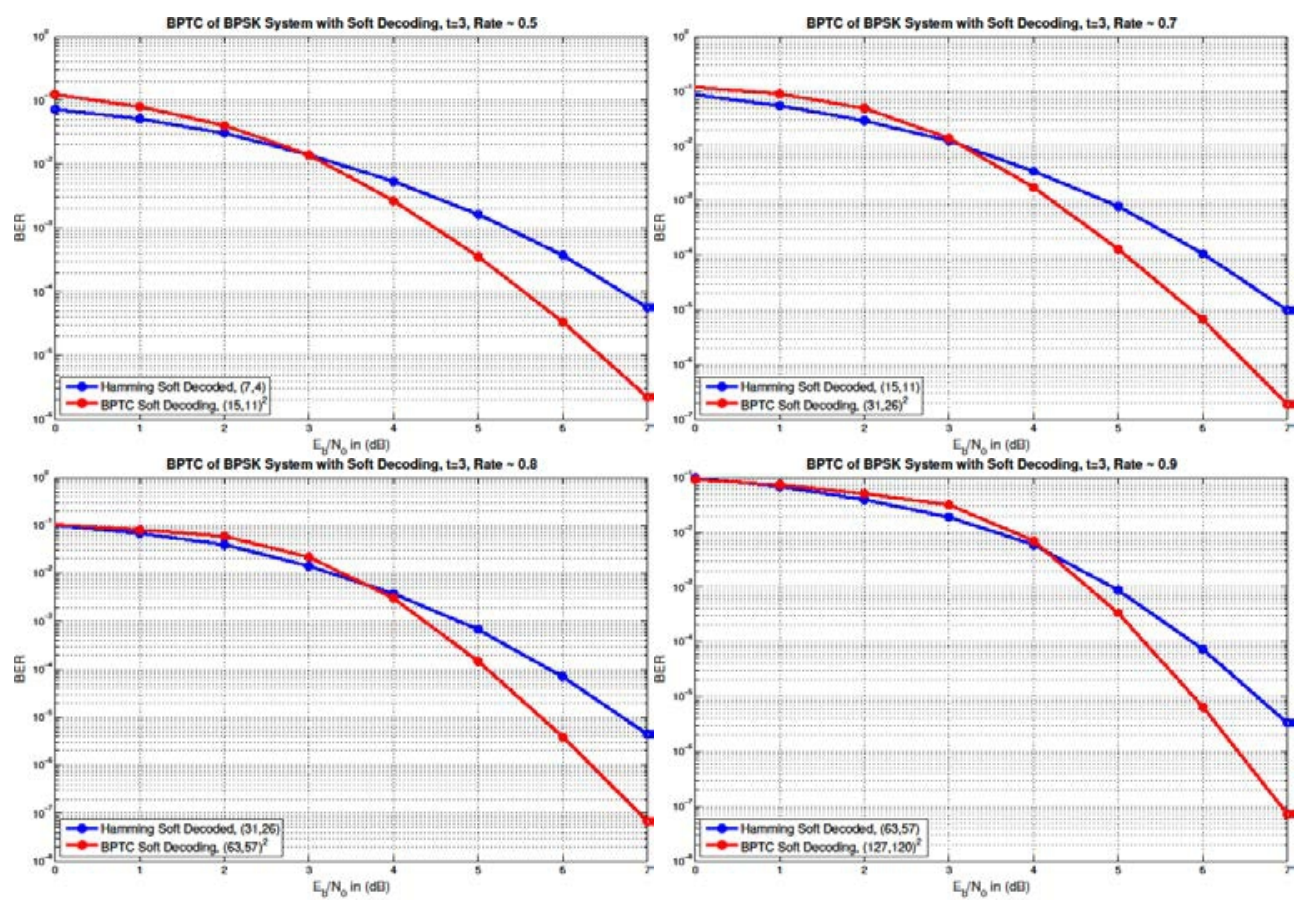

Fig. 15: Simple Hamming code vs. BPTC, with the coding rate.

\section{Conclusion}

In this paper, the design and implementation of the soft decoding algorithm for the Hamming codes with duet and triplet bit error correction. And the structure of the block product turbo code (BPTC) is shown with two different decoding scenarios, the first one is the hard decoding and the second is the soft decoding which use our proposed soft decoder scheme. The simulation results show that the soft decoding of the Hamming codes improves well the performance of the coding by more than $1 \mathrm{~dB}$ in some case. The BPTC scheme decoded hard doesn't improve the performance with respect to the soft decoding of the simple Hamming code, but when our proposed soft decoder is used in the BPTC scheme, we obtained a gain of approximately $1 \mathrm{~dB}$ with respect to the soft decoding of the simple Hamming code. Finally, we have shown a good comparison between different Hamming code size and rate, and we have demonstrated that the $(31,26)$ Hamming code can be a good compromise when concatenated to give a BPTC code.

\section{References:}

Forney, G. D., Jr., Concatenated Codes (Cambridge, MA: MIT Press, 1966). Berrou, C., Glavieux, A., and Thitimajshima, P., "Near Shannon Limit ErrorCorrecting Coding and Decoding: Turbo Codes," IEEE Proceedings of the 
Int. Conf. on Communications, Geneva, Switzerland, May 1993 (ICC ’93), pp. 1064-1070.

Divsalar, D., Dolinar, S., and Pollara, F., "Iterative Turbo Decoder Analysis Based on Density Evolution," IEEE Journal on Selected Areas in Communications, vol. 19, no. 5, pp. 891-907, May 2001.

Divsalar, D., Dolinar, S., "Concatenation of Hamming Codes and Accumulator Codes with High-Order Modulations for High-Speed Decoding,” IPN Progress Report 42-156, February 15, 2004.

Li, J, Narayanan, K. R. and Georghiades, C. N., "Product accumulate codes: a class of codes with near-capacity performance and low decoding complexity", IEEE Trans. Inform. Theory, vol. 50, pp. 31-46, 2004.

Huang, T. D.-H., Chang, C.-Y., Zheng, Y.-X., and Su, Y. T., "Product Codes and Parallel Concatenated Product Codes,” IEEE Wireless Communications and Networking Conference, March 2007 (WCNC 2007).

Muller, B., Holters, M., Zolzer, U., "Low Complexity Soft-Input Soft-Output Hamming Decoder," IEEE proceedings of the 50th FITCE Congress, Palermo, Italy, September 2011 (FITCE 2011), pp. 242-246.

Chen, Y. H., Hsiao, J. H., Liu, P. F., Lin, K. F., "Simulation and Implementation of BPSK BPTC and MSK Golay Code in DSP Chips", Journal of Communications in Information Science and Management Engineering, CISME Vol. 1, No.4, Pages 46-54, 2011.

Cho, J., and Sung, W., "Reduced complexity chase-pyndiah decoding algorithm for turbo product codes", Department of Electrical Engineering Seoul National University, 2011.

Megha, M.S., "Iterative Decoding Algorithm for Turbo Product Codes," International Journal of Innovative Research in Advanced Engineering (IJIRAE) Volume 1 Issue 2, April 2014. 